\title{
3D PRINTING TECHNOLOGY IN ORTHODONTICS - REVIEW OF CURRENT APPLICATIONS
}

\author{
Tomasz Bartkowiak, Agata Walkowiak-Śliziuk \\ Specialist Dental Practice Agata Walkowiak-Śliziuk, Gorzow Wielkopolski, Poland
}

\begin{abstract}
Additive manufacturing is a relatively new technology with rapidly growing range of applications in many fields of medicine and dentistry. The developments of available computer-aided design software and additive manufacturing hardware allowed integrating the technology in orthodontics, and revolutionizing the workflow in orthodontic practices and laboratories around the world. 3D printing is now easily accessible for orthodontists, being a reliable and cost-effective manufacturing method, which may be used in many aspects of orthodontic practice, and its potential is still growing. The article gives necessary insight into the history, development, and available technologies of additive manufacturing. Moreover, it summarizes and reviews current literature concerning all aspects of clinical use of 3D printing in orthodontics.
\end{abstract}

KEY wORDs: 3D printing, additive manufacturing, orthodontics, review.

J Stoma 2018; 71, 4: 356-364

DOI: https://doi.org/10.5114/jos.2018.83410

\section{INTRODUCTION}

Rapid development of digital technologies in dentistry began in 1980s with the implementation of computer-aided design and computer-aided manufacturing (CAD/CAM) in prosthodontics for chairside fabrication of the first ceramic inlay with the Cerec 1 system $[1,2]$. The concept of CAD/CAM requires three functional elements: 1) digitalization tool for data acquisition (a scanner); 2) software to edit and to process digital data; 3) a manufacturing technology to transform digital data to a final product [3]. Since the beginnings of CAD/ CAM in dentistry, the development of the technology has been inherently related to prosthodontics. The first application of CAD/CAM systems was the fabrication of dental restorations. Nowadays, further developments of CAD/CAM allowed for integration of the technology in other fields of dentistry, including maxillofacial surgery, dental implantology, and orthodontics. The main requirement to diversify the range of CAD/CAM applications is the progress in manufacturing technologies. Manufacturing can be held either with subtractive or additive methods. Subtractive fabrication is accomplished by removing material from a block by milling or cutting until a desired shape is created [4]. On the other hand, additive manufacturing is referred to a process, in which an object is fabricated by adding a raw material layer-by-layer in a specific manner $[4,5]$. Other terms for this process used in literature are 3D printing (3DP) and rapid prototyping (RP) $[6,7]$. The aim of the article is to give insight into the development and available $3 \mathrm{D}$ printing technologies, and to review the literature concerning the application of 3D printing in clinical orthodontics.

\section{JOURNAL OF STOMATOLOGY CZASOPISMO STOMATOLOGICZNE}

ADDRESS FOR CORRESPONDENCE: Tomasz Bartkowiak, 6/3 Bp. W. Pluty St., 66-400 Gorzow Wielkopolski, Poland, e-mail: tom.bartkow@gmail.com 


\section{WHAT IS 3D PRINTING? BRIEF HISTORY OF ORIGINS AND DEVELOPMENT}

3D printing was developed in 1980 s by Charles Hull, who created the first 3D printer and 3D printing technology, which was named by the founder as stereolithography [8]. Moreover, Hull created the STL (standard tessellation language or stereolithography) file format. STL file is a triangular representation of an object's surface geometry. Each object is built of many triangles and the peak of each triangle is represented by the coordinates system [9]. STL is the most commonly used file format to edit and prepare the object for 3D printing [8]. The next milestone in the development of 3D printing was the achievement by Scott Crump, who some years following Hull developed a new 3D printing technique called "fused deposition modelling" (FDM) [10]. $3 \mathrm{D}$ printing is a manufacturing technology, in which an object is created by adding a material layer-by-layer one on top of the other in a specific manner, until whole object is manufactured according to computers' design $[5,7]$. The process must be preceded by creating a project/design in STL file format. The digital project is then sliced into layers and the $3 \mathrm{D}$ printer recreates the layers, which are then fused together. Digital design may be created using a computer and dedicated software (CAD), it may be acquired by scanning the structure we want to print, or alternatively, it may be converted to CBCT DICOM file $[8,10]$. To the contrary, subtractive manufacturing involves cutting an object from a material block by removing the excess. $3 \mathrm{D}$ printing is an important step forward in manufacturing technology. Additive manufacturing costs less, allowing high quality production [7, 8]. Additive techniques permit to produce complex and sophisticated shapes and geometries with undercuts or holes, which cannot be manufactured by any other available method $[11,12]$. A variety of materials may be applied to print objects with different 3D printers, including gypsum, metal alloys, glass, carbon fiber, resins as well as organic materials, living cells, and tissues. Some of above-mentioned materials have been used in dentistry and orthodontics. Developments in the field of printers and materials set the basis for the use of the technology in industry (e.g., automobile, aviation, aerospace, defense), science, and medicine.

\section{D PRINTING - APPLICATIONS IN MEDICINE AND DENTISTRY}

The most commonly cited application of 3D printing in medicine is fabrication of different surgical stents [12, 13]. $3 \mathrm{D}$ printed surgical stents have been reported to be used in orthopedics, general and oncological surgery as well as cardio- and neurosurgery. 3D printed models facilitate diagnostic processes through three-dimensional recreation of anatomical structures, thus allowing to plan the surgical intervention in detail [14]. The technology has also been applied to fabricate customized implants of organs and tissues recreating, damaged or removed anatomical structures, like cranium calvaria, ectoprostheses, or ear implants [13]. 3D printing is also used to manufacture pharmaceuticals [15]. One of the newest fields of 3D printing application in medical setting is bioprinting, i.e., the 3D printing technology using cells and tissues [16]. Developments of technologies and materials allowed to integrate $3 \mathrm{D}$ printing in dentistry. Applications of $3 \mathrm{D}$ printing in dentistry reported in the literature include manufacturing of surgical stents for guided implant positioning [17]. Moreover, researchers attempted to fabricate customized dental implants from titanium alloys and zirconium with additive technologies $[18,19]$. A recently developed and reported application of 3D printing with biomaterials has taken place in the field of regenerative periodontal surgery to manufacture resorbable scaffolds, such as hydrogels [20]. Maxillofacial surgery has also profited from integrating $3 \mathrm{D}$ printing; it has been used to reconstruct bony structures of maxilla and mandible damaged by trauma or neoplasm by fabricating metal plates and customized implants $[11,21]$. Orthognathic procedures have also been a target for $3 \mathrm{D}$ printing application, since orthognathic surgery wafers may be fabricated by additive technologies $[11,13,22]$. Likewise, in case of general surgery, 3D printing of anatomical models serves as an important aid for maxillofacial surgeons in thorough surgery planning, which has been reported to result in reduced surgery time and improved functional and esthetic treatment outcomes [13, 21]. On the other hand, in prosthodontics, 3D printing has been applied to fabricate individual impression trays, metal frameworks for removable dentures, fixed metal copings, mockups, and resin patterns for further conventional metal castings [22]. The application of 3D printing in orthodontics is presented below.

\section{D PRINTING TECHNOLOGIES}

$3 \mathrm{D}$ printing is a general term referring to diverse technologies, which are listed and described below that have been reported to be used in orthodontics.

\section{STEREOLITHOGRAPHY}

Stereolithography (SLA) was conceived and patented by the founder of $3 \mathrm{D}$ printing, Charles Hull, being the first 3D printing technology that has been developed [8]. Stereolithography employs photocurable liquid resin as a printing material (photopolymer). Single layer of a printed object is being selectively hardened by a spot of ultraviolet laser beam, which initiates cross-linking of the polymer and thus solidifies the material. Following curing, resin is being spread on 
top of the previous layer and the cycle is repeated until the object is constructed [7, 23]. Stereolithography requires certain procedures of post-processing the object after printing. The unpolymerized resin has to be removed and the object needs to undergo a post-curing process in a UV oven to increase the conversion degree of the polymer [23]. Printing objects with complex shapes (with undercuts or cantilevers) requires incorporating support structures in the CAD design, which have to be removed after printing is completed [7]. Advantages of stereolithography cited in the literature include high quality and high resolution of printed objects, with layer thickness of $25 \mu \mathrm{m}[23,24]$. Low thickness of a layer is a crucial factor to ensure smooth surface of printed object without stair-stepping, resulting from inaccuracies in binding adjacent layers [25]. Stereolithography has been reported to be used for manufacturing of surgical guides in dental implantology [17]. In orthodontics, the technology has been applied to manufacture, among others, orthodontics dental models [25].

\section{FUSED DEPOSITION MODELLING/FUSED FILAMENT FABRICATION}

Nowadays, fused deposition modelling/fused filament fabrication (FDM/FFF) technology is the most commonly used 3D printing method worldwide [10]. The technology employs thermoplastic materials to build solid objects. Thermoplastic material is heated until melting temperature is reached, which is followed by deposition of melted material through extrusion heads by the printer in a specific pattern, creating a single layer of an object. The material is applied layer-by-layer, which are fused together as the material solidifies [23]. The key advantage of fused deposition modelling is that the technology requires no post-processing of the object following printing and enables to print with minimal layer thickness of $127 \mu \mathrm{m}$ [24]. A certain disadvantage and limitation of this technology results from material shrinkage during hardening, and the necessity to use materials with sufficient thermal and viscoplastic properties. Available materials for FDM include acrylonitrile butadiene styrene polymer (ABS), polycarbonates (PCA), polylactic acid (PLA), waxes, and polyphenylsulfones [26]. An example of FDM 3D printer with application in orthodontics is Makerbot Replicator2 (Stratasys Ltd., Eden Prairie, MI). According to the manufacturer's data, the printer may be used to fabricate retainers and aligners, although with inferior esthetic quality due to stair-stepping effect [26].

\section{SELECTIVE LASER SINTERING/MELTING AND ELECTRON BEAM MELTING}

Selective laser sintering (SLS) employs powder materials to print $3 \mathrm{D}$ objects [26]. A high energy $\mathrm{CO}_{2}$ laser beam is used to heat the powder above the glass transition temperature in a specific pattern, and thus sinter the particles together [23]. After completion of a single layer, the printer building platform lowers, and another layer of powder is added on the previous one and sintering continues until the printing of the object is completed [7]. SLS does not require any support structures to be incorporated into the design because partially sintered unbound powder particles ensure sufficient support for complex geometries of a printed object. Following 3D printing, removal of the excess of powder particles is necessary, being a relatively simple procedure [26]. Powder materials, which may be applied for SLS technology printing include polymers like polyamides, polycaprolactone, hydroxyapatite, glass, ceramics, and powdered metallic alloys such as stainless steel, titanium, and $\mathrm{Co} / \mathrm{Cr}$ [26]. Electron beam melting (EBM) technology also uses metal powder as printing material. The powder undergoes sintering by computer-controlled electron beam in vacuum, unlike in SLS, which employs laser beam. Metal alloys used in EBM are stainless steel, titanium, and cooper [26]. Structures printed using EBM are highly porous and mechanically strong, therefore EBM technology has been used in orthopedics and oral surgery to fabricate customized osseointegrated implants $[23,26]$.

\section{DIGITAL LIGHT PROCESSING}

Digital light processing (DLP) is similar to stereolithography and uses photopolymer as a printing material. Photopolymer is cured by light with the use of digital micromirror device (DMD). The main difference to stereolithography is the fact that the light is projected on the whole layer, while stereolithography employs spot laser beam. DLP enables faster printing than stereolithography, with layer thickness of less than $30 \mu \mathrm{m}$ [26, 27]. EnvisionTEC (Gladbeck, Germany) introduced 3D printers applying the following technology to the market: Ultra 3SP Ortho and Perfactory Micro Ortho. According to the manufacturer, Ultra 3SP printer is designed to manufacture accurate dental working models for orthodontic appliance fabrication $[26,28]$.

\section{INKJET 3D PRINTING AND POLYJET}

Inkjet $3 \mathrm{D}$ printing (3DP/IJP) allows manufacturing structures by adding a pattern of binder liquid on a powder substrate. The object is created as a result of a reaction between liquid and powder, while phase transformation is caused by ultraviolet curing, chemical or thermal reaction, or by dehydration [26]. In the following technology, various materials may be used during one printing cycle allowing to fabricate objects with different materials, thus exhibiting diverse properties [23].Thickness of a layer has been reported to be up to 
$12 \mu \mathrm{m}$ [24].3DP is faster than FDM, but surface finish and accuracy may be inferior than those achieved with stereolithography [26]. Further developments in the technology have led to create PolyJet (polymer jetting) by Stratasys Ltd. (Eden Praire, USA). PolyJet employs liquid photopolymer as a printing material. The resin is spread by a nozzle on a building platform, which is subsequently cured with ultraviolet light. Supportive structures are created with layers of photopolymeric material. Supporting material may be removed easily after completion of printing process. To the contrary to stereolithography, an object printed with PolyJet is cured maximally and requires no additional post-curing. Layer thickness achieved by PolyJet printers ranges from $16 \mu \mathrm{m}$ to $32 \mu \mathrm{m}$, which makes this technology accurate $[26,29]$. PolyJet printers that are commercially available include Objet30 OrthoDesk, Objet30 Dental Prime, and Objet500 Dental Selection (Stratasys Ltd., Eden Praire, USA) [29]. According to the manufacturer's data, Objet30 OrthoDesk printer may be used to manufacture orthodontic study and working models, surgical stents and dental mockup, depending on the printing material $[26,29]$.

\section{D PRINTING IN ORTHODONTICS}

\section{DIAGNOSTIC AND WORKING ORTHODONTIC MODELS}

3D printing technology may be used in orthodontics to manufacture models of patients' dentition. Increasing popularity and growing application of intra- and extraoral scanners and digital dental models, contributes to a significant decrease in a need to acquire alginate impressions and casting plaster models, thereby allowing avoiding drawbacks of conventional orthodontic models. Digital models may be used for orthodontic diagnostic purposes. Diagnostic measurements performed on digital models represent high validity, reliability, and reproducibility, and thus may be regarded as an equal alternative to conventional plaster models [30-32]. Although in cases, in which manufacturing of orthodontic appliances is planned, a physical model of patient's dentition is required. 3D printing enables to transform digital, virtual dental model of patient's dentition into a physical model, omitting certain steps, which are conventionally required, including impression taking and model casting [33, 34]. Moreover, rapid prototyping technology allows to manufacture many identical copies of a digital model without any risk of distortion or deformation, being available at any time [34]. Printed models have been reported and may be used to manufacture removable orthodontic appliances, expansion appliances, indirect bonding trays, or thermoformable orthodontic aligners [35, 36]. $3 \mathrm{D}$ printing technology has set the basis for the use and rapid developments in the field of aligners. Manufacturing of a set of aligners for a single patient requires to perform individual digital setup, which allows to plan movements of teeth during treatment. A sequence of models, each one reflecting one single stage of treatment, needs to be fabricated to allow producing a set of aligners [37]. Moreover, the range of tooth movement performed by an aligner ranges between $0.25 \mathrm{~mm}$ to $0.30 \mathrm{~mm}$, thus indicating the requirements concerning the accuracy of working model fabrication [38]. Any inaccuracies in the printing process of working models may result in inadequate tooth movements, negatively influencing treatment outcomes. In the case of diagnostic models, inaccurate diagnostic records may be taken and impeding crucial treatment planning decisions. Therefore, the application of each 3D printing technology to manufacture dental models needs to be thoroughly considered regarding printing accuracy and availability of materials. Direct comparison of 3D printing technologies can only give answers which technology is appropriate for a specific orthodontic application.

Kim et al. [34] investigated precision and trueness of selected diagnostic measurements performed on scans of models, which were printed with 4 technologies including SLA, PolyJet, DLP, and FFF. The results of the study revealed statistically significant differences between measurements taken on models printed with all 4 methods. Measurements on PolyJet and DLP models had higher precision than for SLA and FFF models. On the other hand, trueness was reported to be highest for stereolithographic models [34]. Different approach to research into the accuracy of diagnostic measurements on $3 \mathrm{D}$ printed models has been presented by Wan Hassan et al. [39]. The authors compared selected measurements performed directly on printed models obtained from Z printer (Stratasys Ltd., Eden Praire, USA) to conventional plaster models using digital caliper, depending on the severity of crowding. For each group of models with a specific severity of crowding (mild/moderate/ severe), there were statistically significant differences for tooth widths, tooth heights, and arch width measurements. According to Wan Hassan et al., the findings excluded the clinical use of printed models in orthodontic diagnosis and treatment planning [39]. The reason for the results was not sufficient printing accuracy in recreating anatomical details, which negatively influenced the ability to identify landmarks for measurements [39]. Dietrich et al. [40] examined precision and trueness of stereolithographic and PlyJet printed models. To perform the analysis, the models were scanned following printing and then superimposed using best-fit algorithm in a special software. Trueness was reported to be higher for PolyJet models, but precision was significantly higher for models printed with stereolithography $(p<0.05)$. Maximal surface deviations for both groups were reported not to exceed $127 \mu \mathrm{m}$, which makes both 
printing technologies acceptable for orthodontic diagnostics [40]. Hazeveld et al. [41] on the other hand compared selected linear measurements performed on conventional plaster and printed models using DLP, PolyJet, and 3DP technologies. Bland-Altman plots indicated that the agreement was high between tooth width and crown height measurements for PolyJet and DLP models, with mean systematic differences $-0.08 \mathrm{~mm}$ for PolyJet and $-0.05 \mathrm{~mm}$ for DLP. According to the authors and other studies, the threshold of clinical relevance in measurements is $0.3 \mathrm{~mm}$, which makes both technologies acceptable regarding the accuracy in clinical orthodontics [41, 42]. Camardella et al. [43] investigated the influence of the design of a model base on the accuracy of models printed with stereolithography and PolyJet technology. The researchers assessed 3 types of model bases: regular ABO (American Board of Orthodontics) base, horseshoe-shaped based, and horseshoe-shaped base with a transverse bar. Horseshoe-shaped bases are frequently designed in models used to manufacture thermoformable orthodontic aligners, thus the assessment of their accuracy is of significant clinical importance [44]. The results have shown that models with a horseshoe-shaped base with a bar and regular base were accurate, regardless of $3 \mathrm{D}$ printing technology used. Models with a horseshoe-shaped base without a bar, which were printed using stereolithography were characterized by statistically significant reduction in transverse dimension in comparison to models with regular base $(p=0.000)$. As a reason for these findings, the authors named the post-curing process of stereolithographic models, which in the absence of a transverse supporting bar, could result in material shrinkage and decrease of posterior region dimension [43]. These differences were not observed for PolyJet printed models.

Available literature concerning 3D printed models report many other interesting applications, which include visualization, diagnosis, and treatment planning. One of the papers has been published by Lee et al. [45]. The authors described a technique of 3D printing teeth replica for autotransplantation. 3D printed teeth replica permits for shortening and simplifying the procedure, minimizing the risk of damage to the transplant tooth during checking for fit in the prepared site and finally, contributing to shortening the extraoral time of the transplant tooth $[45,46]$. An important factor limiting the use of printed teeth replica is the accuracy of additive manufacturing technology. Lee et al. [45] investigated 50 teeth replicas printed with PolyJet and FDM technologies. The results indicate that there were statistically significant differences in accuracy of teeth replica depending on the printing technology $(p<0.05)$ but according to the authors, the differences were not clinically relevant, allowing to apply both technologies for the following purpose [45]. Also, Faber et al. [47] implemented 3D printing into orthodontic diagnosis and decision making. The authors described 3D printing of an anatomical model obtained on the basis of computed tomography images of an impacted maxillary canine. The printed dimensional model enabled the clinicians to conduct thorough assessment and visualization of the anatomy and localization of the impacted tooth, also in relations to the roots of adjacent teeth. Model analysis helped the authors to precisely plan the surgical exposure procedure of the impacted tooth [47].

\section{REMOVABLE ORTHODONTIC RETAINERS}

Computer-aided design and 3D printing open new possibilities in orthodontics to manufacture customized removable retainers. The procedure has been presented and described by Nasef et al. [48]. The process integrates the application of new technologies, including cone beam computed tomography (CBCT), $\mathrm{CAD}$ and $3 \mathrm{D}$ printing. The first step in the procedure is scanning patient's dentition using CBCT and image conversion into a STL file to create a 3D model of patient's dentition. Following importing the file into dedicated software (Zbrush 4R4, Pixologic, Los Angeles, California), the retainer is designed virtually. The virtual project representing the retainer is (upon acceptance) manufactured by printing. Nasef et al. [48] used Formiga P100 3D printer (EOS, Munich, Germany). The printer applies SLS technology. The printing material used was a fine polyamide PA 2200 (EOS). A certain disadvantage of the material used may be its white opaque color. The application of stereolithography technique would allow to use another printing material, achieving an ideal transparency. Another disadvantage of SLS, named by authors, are high costs and still low availability. However, according to the authors, the described method may be successfully used with other 3D printing technologies and materials. Nasef et al. [48] did not mention or describe any clinical nor scientific evaluation of the printed retainer in their article.

\section{REMOVABLE ORTHODONTIC APPLIANCES}

First trials to manufacture removable acrylic orthodontic appliances using computer-aided design and 3D printing have been made and presented by Sassani et al. [49]. The authors reported the application of halfautomated technique to manufacture acrylic base plates of removable appliances. A machine dedicated for this particular purpose has been used to add and polymerize layers of acrylic, which were added according to the computer design of the appliance. The screws and wires however needed to be placed manually onto the working model, their incorporation in the virtual design and manufacturing process has been reported not to be possible at that time [49]. Al Mortadi et al. [50] described a procedure of Adresen activator and sleep 
apnea appliance fabrication using computer-aided design and additive manufacturing technology. The first step in the procedure was digitalization of plaster models of patient's dentition using a laser scanner. Construction bite and virtual appliance design was made using CAD software (FreeForm Modeling Plus, version 11; Geo Magics SensAble Group, Wilmington, Mass) in conjunction with special phantom (haptic) arm (Geo Magics SensAble Group). The acrylic baseplate of the appliance has been designed. The design involved modeling of a palatal plate, bite blocks covering occlusal surfaces of mandibular, and maxillary teeth to form a monoblock and anterior capping covering lower incisors. The labial bow was bent manually in a conventional way, with $0.9 \mathrm{~mm}$ stainless steel wire. To incorporate the labial bow into the acrylic, the authors designed special guiding jigs, which enabled precise positioning of the wire in the acrylic plate. Manufacturing process of the virtually designed activator was held using stereolithography machine (SLA 250-50; 3D Systems). Following printing, the appliance was cleaned in isopropanol solvent (99\%) and support structures were removed. Post-curing was achieved by ultraviolet light polymerization to increase the degree of polymer conversion. According to the authors, the manufacturing process with stereolithography has been chosen because of the possibility to pause printing, which was necessary to attach conventionally bent labial bow [50]. The authors conducted clinical evaluation of the appliance and stated that the fit and adaptation to the model surface were satisfactory, both palatially and lingually [50]. The appliance surface was smooth, with no sharp edges. Labial bow was fitted firmly into the plate and was functional [50].

The next development in the field was fabrication of Hawley retainer with CAD and 3D printing. Al Mortadi et al. [51] presented Hawley retainer manufacturing using intraoral scans obtained with TRIOS (3Shape, Copenhagen, Denmark), eliminating the need of conventional impression taking and pouring plaster models. During the stage of creating virtual appliance, the shape, thickness and range of acrylic base plate, fitted labial bow, and Adams clasps was designed. Wire elements were bent using cobalt-chromium alloy with $3 \mathrm{D}$ printing technology. The base plate of Hawley retainer was fabricated form ClearVue resin material (3D Systems), implementing stereolithography. In clinical assessment of the retainer, the authors concluded that the quality of the appliance was satisfactory [51]. A disadvantage of the described procedure is the necessity to use complex software and haptic phantom arm, which significantly increases cost of the procedure. On the other hand, strictly controlled manufacturing process is reliable and repeatable, allowing to create appliances with planned thickness, range, and shape [51]. Nowadays, 3D printing technology allows to manufacture wire elements, including labial bows and clasps form metal alloys and to incorporate those parts into the base plate of the appli- ance [51]. Another application of additive manufacturing was to fabricate soft customized, silicone removable appliances introduced by Salmi et al. [52]. The authors printed the appliance using stereolithography (SLA 350 machine - 3D Systems), which was preceded by creating the digital design. Manufactured silicone appliance was subjected to evaluation, which was conducted by scanning the appliance, creating its virtual model, and digital image superimposition on computer-aided design. Maximal deviation of $1 \mathrm{~mm}$ was observed on sharp edges and thin walls of the appliance [52]. According to the authors, the technology used enables faster production, limits the costs, and results in fabricating appliances with high accuracy [52].

The articles cited above are however only case reports, showing the potential of 3D printing application in removable appliance manufacturing process. The methods and techniques described by the authors of the articles need to be assessed, especially concerning costs, workflow, accuracy, and clinical efficiency. So far, literature does not present such data.

\section{CUSTOMIZED LINGUAL ORTHODONTIC BRACKETS AND HERBST APPLIANCE}

Nowadays, 3D printing is used in the process of manufacturing fixed orthodontic appliances. Additive manufacturing is a part of production process of lingual orthodontic brackets. Wiechmann et al. [53] introduced 3D printing to create wax patterns of lingual orthodontic brackets, allowing to customize the shape of bracket base. The manufacturing process begins with virtual design of each bracket, which can be customized to fit ideally to the anatomy of lingual/palatal surface of teeth. Digital design allows to customize in - out, angulation and torque values of each bracket; thus, an individual bracket prescription is created for each patient [53]. The next step employs rapid prototyping to transform virtually designed brackets into wax pattern. The prototypes are then cast in gold to produce final customized brackets that may be used in treatment [53]. Wiechmann et al. [54] in their article described a case report incorporating Herbst appliance in lingual orthodontic therapy. In order to achieve that, 3D printing technology was employed. Digital design of lingual orthodontic brackets involved creating thicker and extended bracket bases on maxillary first molars and mandibular canines to manufacture those brackets as bands. Pivots and tubes were attached to the bands, and proper tube and telescope length as well as design were planned to achieve class I occlusion. Recreation of the designed appliance requires accurate prototyping, which is achieved with $3 \mathrm{D}$ printing technology, allowing (according to the authors) to customize fixed functional therapy and combine it with lingual orthodontic treatment protocol [54]. 


\section{OCCLUSAL SPLINTS}

Occlusal splints are contemporarily used for treatment of patients presenting with temporomandibular disorders (TMD). The conventional process of splints fabrication in dental laboratory requires taking alginate impressions of patient's dentition, wax bite registration, and mounting casts in articulator. Lauren and McIntyre were the first authors to publish an article, which describes digital workflow in occlusal splints manufacturing [55]. The suggested digital protocol applied subtractive technology of splint fabrication, which were machined down from acrylic material block. Salmi et al. [56] introduced 3D printing into the process of splint manufacturing. Occlusal splints were made by the authors using stereolithography machine SLA 350 (3D Systems, USA). 3D printed splint has been evaluated clinically after 1,3 , and 6 months of patient's use. The adaptation process to splint therapy has been positive and muscle tension has been relieved. No signs of tooth or splint wear has been detected after 6 months of clinical use [56]. Moreover, the splint has been thoroughly tested following scanning and the scan was superimposed onto splint virtual design with special software. Inaccuracies in splint dimensions and surface deviations of $1 \mathrm{~mm}$ were reported at the edges and sharp margins of the splint; however, maximal deviations in other parts of the appliance did not exceed $0.3 \mathrm{~mm}$ [56]. These findings indicate that $3 \mathrm{D}$ printing has potential to become routinely used in occlusal splints manufacturing. The printing process is highly reproducible and faster than conventional technique, thus decreases significantly the dental laboratory workload. According to the authors, improved 3D printed splint accuracy may reduce time required to trim the splint $[56,57]$. On the other hand, there is still a need for further clinical and scientific examination of $3 \mathrm{D}$ printed occlusal splints concerning clinical use.

\section{SURGICAL TEMPLATES FOR ORTHODONTIC MINISCREWS AND MINIPLATES PLACEMENT}

Orthodontic miniscrews and miniplates are used as a source of intraoral maximal anchorage. Loading of miniscrews or plates allows to minimize reaction forces acting on teeth and also, broadens the scope of possible tooth movements [57]. Regarding force control, miniscrew stability and anatomical limitations including limited space for miniscrew placement, risk of root damage, perforation of maxillary sinus or neurovascular damage in miniscrew insertion, a factor of crucial importance for correct miniscrew positioning [58]. Various techniques have been developed to ensure proper miniscrew placement trajectory and localization, but according to the contemporary research data, the following methods do not guarantee sufficient precision $[59,60]$.
Wang et al. described a technique of orthodontic miniscrew placement using a 3D printed surgical template [61]. The authors applied superimposed CBCT and dentition scan data, which were imported into CAD software to design virtual surgical template. The template was $3 \mathrm{D}$ printed with ABS material (acrylonitrile butadiene styrene) using FDM 3D printing machine. The template was subjected to clinical evaluation following fabrication. The template had a good fit to the surface of oral mucosa (mean gap size of $0.3 \mathrm{~mm}$ ), good holding power, and fitting adoption to patient's teeth, ensuring sufficient stability during miniscrew insertion. In order to manufacture surgical templates with high accuracy and ability of intraoral application, it is necessary to use high resolution 3D printers and biocompatible printing materials. Time and workload required to design and manufacture the template need to be considered in routine clinical use [61].

Miniplates may be used as well as a source of maximal anchorage in orthodontics. Miniplates are a certain alternative to orthodontic miniscrews, their range of applications include different orthodontic treatment procedures such as maxillary molars intrusion, open bite treatment, maxillary molar distalization, or maxillary protraction or impaction $[62,63]$. Placing a miniscrew requires surgical procedure to secure the plate with screws to the bone surface. Precise miniplate placement and good adoption to the bone surface allows to decrease failure rates, and enables the orthodontist to apply required mechanics. Hourfar et al. [64] introduced a method of customized adaptation and placement of orthodontic miniplates using $\mathrm{CAD}$ and $3 \mathrm{D}$ printing technology. 3D printed model of patient's bone fabricated on the basis of CBCT images has been used as a template to position and adopt the miniplate to the bony contour on the model. Stereolithographic 3D printer has been used to manufacture the model. Miniplate, which final position has been established and fixed with screws, served as a guide to fabricate a jig to transfer the plate to the patient's mouth. According to the authors, the main advantage of this technique is precise determination of the final position of an orthodontic miniplate prior to the surgical procedure, which significantly reduces the time needed for surgery and simplifies the process [64]. Another advantage results from pre-operative adaptation of the plate to the bone surface, allowing for maximum contact between the miniplate and the bone, and decreasing the possibility of miniplate failure [64].

\section{CONCLUSIONS}

3D printing technology has become more widely used in orthodontics and the scope of possible applications is still expanding. Available literature gives many examples of various $3 \mathrm{D}$ printing techniques and materials in a wide range of applications. Acceptance of 3D printing technology and material used in a certain clinical situa- 
tion depends on building a broad basis of scientific evidence from both in-vitro and clinical trials, which allows to draw conclusions concerning accuracy, costs, clinical efficiency, and further potential new methods. Scientific and manufacturers' recommendations concerning applications of a certain technology are to be followed strictly in order to minimize risks and help to achieve clinical success.

\section{CONFLICT OF INTEREST}

The authors declare no potential conflicts of interest with respect to the research, authorship, and/or publication of this article.

\section{References}

1. Miyzaki T, Hotta Y. CAD/CAM systems available for the fabrication of crown and bridge restorations. Aust Dent J 2011; 56 (1 Suppl): 97-106.

2. Mörmann WH. The evolution of CEREC system. J Am Dent Assoc 2006; 137 Suppl: 7-13.

3. Beuer F, Schweiger J, Edelhoff D. Digital dentistry: an overview of recent develompents for CAD/CAM generated restorations. Br Dent J 2008; 204: 505-511.

4. Bae EJ, Jeong ID, Kim WC, Kim JH. A comparative study of additive and subtractive manufacturing for dental restorations. J Prosthet Dent 2017; 118: 187-193.

5. Andonovic V, Vrtanoski G. Growing rapid prototyping as a technology in dental medicine. Mech Eng Sci J 2010; 29: 31-39.

6. Beguma Z, Chhedat P. Rapid prototyping - when virtual meets reality. Int J Comput Dent 2014; 17: 297-306.

7. Ligon SC, Liska R, Stampfl J, et al. Polymers for 3D printing and customized additive manufacturing. Chem Rev 2017; 117: 10212 10290.

8. Dodziuk H. Applications od 3D printing in healthcare. Kardiochir Torakochir Pol 2016; 13: 283-293.

9. Richert R, Goujat A, Venet L, et al. Intraoral Scanner Technologies: A Review to Make a Successful Impression. J Healthc Eng 2017 2017: 8427595.

10. Ludwikowski F. Technologie druku 3D. Elektronika Praktyczna 2017; 4.

11. Bhargav A, Sanjairaj V, Rosa V, et al. Applications of additive manufacturing in dentistry: a review. J Biomed Mater Res B Appl Biomater 2018; 106: 2058-2064

12. Liaw CY, Guvendiren M. Current and emerging applications of 3D printing in medicine. Biofabrication 2017; 9: 024102.

13. Tack P, Victor J, Gemmel P, Annemans L. 3D-printing techniques in a medical setting: a systematic literature review. BioMed Eng OnLine 2016; 15: 115 .

14. Pucci JU, Cristophe BR, Sisti JA, Connolly ES Jr. Three-dimensional printing: technologies, applications, and limitations in neurosurgery. Biotechnol Adv 2017; 35: 521-529.

15. Alhnan MA, Okwuosa TC, Sadia M, et al. Emergence of 3D printed dosage forms: opportunities and challenges. Pharm Res 2016; 33: 1817-1832.

16. Tappa K, Jammalamadaka U. Novel biomaterials used in medical 3D printing techniques. J Funct Biomater 2018; 9: 17.

17. Neumeister A, Schultz L, Glodecki C. Investigations on the accuracy of 3D-printed drill guides for dental implantology. Int J Comput Dent 2017; 20: 35-51.

18. Tunchel S, Blay A, Kolerman R, et al. 3D Printing/additive manufacturing single titanium dental implants: a prospective multicenter study with 3 years of follow-up. Int J Dent 2016; 2016: 8590971.
19. Osman RB, van der Veen AJ, Huiberts D, et al. 3D-printing zirconia implants; a dream or a reality? An in-vitro study evaluating the dimensional accuracy, surface topography and mechanical properties of printed zirconia implant and discs. J Mech Behav Biomed Mater 2017; 75: 521-528.

20. Larsson L, Decker AM, Nibali L, et al. Regenerative medicine for periodontal and peri-implant diseases. J Dent Res 2016; 95: 255-266.

21. Matias M, Zenha H, Costa H. Three-dimensional printing: custom-made implants for craniomaxillofacial reconstructive surgery. Cranomaxillofac Trauma Reconstr 2017; 10: 89-98.

22. Alharbi N, Wismeijer D, Osman RB. Additive manufacturing techniques in prosthodontics: where do we currently stand? a critical review. Int J Prosthodont 2017; 30: 474-484.

23. Chia $\mathrm{HN}, \mathrm{Wu} \mathrm{BM}$. Recent advances in $3 \mathrm{D}$ printing of biomaterials. J Biol Eng 2015; 9: 4.

24. Liu Q, Leu MC, Schmitt SM. Rapid prototyping in dentistry: technology and application. Int J Advan Manu Tech 2005; 29: 317-335.

25. Barazanchi A, Li KC, Al-Almeh B, et al. Additive technology: update on current materials and applications in dentistry. J Prosthod 2017; 26: 156-163

26. Taneva E, Kusnoto B, Evans CA. 3D scanning, imaging, and printing in orthodontics. In: Issues in Contemporary Orthodontics. Bourzgui F (ed.). InTech. Available from: https://mts.intechopen. com/books/issues-in-contemporary-orthodontics/3d-scanning -imaging-and-printing-in-orthodontics

27. Osman RB, Alharbi N, Wismeijer D. Build angle: does it influence the accuracy of 3D-printed dental restorations using digital light-processing technology. Int J Prosthodont 2017; 30: 182-188. 28. https://envisiontec.com/ (Accessed: 7.02.2018).

29. http://www.stratasys.com/polyjet-technology/ (Accessed: 14.02.2018).

30. Naidu D, Freer TJ. Validity, reliability, and reproducibility of the iOC intraoral scanner: A comparison of tooth widths and Bolton ratios. Am J Orthod Dentofacial Orthop 2012; 144: 304-310.

31. Nalcaci R, Topocuoglu T, Ozturk F. Comparison of Bolton analysis and tooth size measurements obtained using conventional and three - dimensional orthodontic models. Eur J Dent 2013; 7: 66-70.

32. Fleming PS, Marinho V, Johal A. Orthodontic measurements on digital study models compared with plaster models: a systematic review. Orthod Craniofac Res 2011; 14: 1-16.

33. Kasparova M, Grafova L, Dvorak P, et al. Possibility of reconstruction of dental plaster cast from 3D digital study models. Biomed Eng Online 2013; 12: 49.

34. Kim SY, Shin YS, Jung HD, et al. Precision and trueness of dental models manufactured with different 3-dimensional printing techniques. Am J Orthod Dentofacial Orthop 2018; 153: 144-153.

35. Ciuffolo F, Epifania E, Duranti G, et al. Rapid prototyping: a new method of preparing trays for indirect bonding. Am J Orthod Dentafacial Orthop 2006; 129: 75-77.

36. Martorelli M, Gerbino S, Giudice M, Ausiello P. A comparison between customized clear and removable orthodontic appliances manufactured using RP and CNC techniques. Dent Mater 2013; 29: e1-10.

37. Malik OH, McMullin A, Waring DT. Invisible orthodontics part 1 : Invisalign. Dent Update 2013; 40: 203-204.

38. Phan X, Ling PH. Clinical limitations of Invisalign. J Can Dent Assoc 2007; 73: 263-266.

39. Wan Hassan WN, Yusoff Y, Mardi NA. Comparison of reconstructed rapid prototyping models produced by 3 -dimensional printing and conventional stone models with different degrees of crowding. Am J Orthod Dentofacial Orthop 2017; 151: 209-218.

40. Dietrich CA, Ender A, Baumgartner S, Mehl A. A validation study of reconstructed rapid prototyping models produced by two technologies. Angle Orthod 2017; 87: 782-787.

41. Hazeveld A, Huddleston Slater JJR, Ren Y. Accuracy and reproducibility of dental replica models reconstructed by different rapid prototyping techniques. Am J Orthod Dentofacial Orthop 2014; 145: 108-115.

42. Hirogaki Y, Sohmura T, Satoh H, et al. Complete 3-D reconstruction of dental cast shape using perceptual grouping. IEEE Trans Med Imaging 2001; 20: 1093-1101. 
43. Camardella LT, de Vasconcellos Vilella O, Breuning H. Accuracy of printed dental models made with 2 prototype technologies and different designs of model bases. A J Orthod Dentofacial Orthop 2017; 151: 1178-1187.

44. Kuo E, Miller RJ. Automated custom - manufacturing technology in orthodontics. Am J Orthod Dentofacial Orthop 2003; 123: 578-581.

45. Lee KY, Cho JW, Chang NY, et al. Accuracy of three-dimensional printing for manufacturing replica teeth. Korean J Orthod 2015; 45: 217-225.

46. Verweij JP, Jongkees FA, Anssari Moin D, et al. Autotransplantation of teeth using computer-aided rapid prototyping of a threedimensional replica of the donor tooth: a systematic literature review. Int J Oral Maxillofac Surg 2017; 46: 1466-1474.

47. Faber J, Berto PM, Quaresma M. Rapid prototyping as a tool for diagnosis and treatment planning for maxillary canine impaction. Am J Orthod Dentofacial Orthop 2006; 129: 583-589.

48. Nasef AA, El-Beialy AR, Mostafa YA. Virtual techniques for designing and fabricating a retainer. Am J Orthod Dentofacial Orthop 2014; 146: 394-398.

49. Sassani F, Elmajian A, Roberts S. Computer-assisted fabrication of orthodontic appliances: considering the possibilities. J Am Dent Assoc 1995; 126: 1296-1300.

50. Al Mortadi N, Eggbeer D, Lewis J, Williams RJ. CAD/CAM/AM applications in the manufacture of dental appliances. Am J Orthod Dentofacial Orthop 2012; 142: 727-733.

51. Al Mortadi N, Jones Q, Eggbeer D, et al. Fabrication of a resin appliance with alloy components using digital technology without an analog impression. Am J Orthod Dentofacial Orthop 2015; 148: 862-867.

52. Salmi M, Tuomi J, Sirkkanen R, et al. Rapid tooling method for soft customized removable oral appliances. Open Dent J 2012; 6: 85-89.

53. Wiechmann D, Rummel V, Thalheim A, et al. Customized brackets and archwires for lingual orthodontic treatment. Am J Orthod Dentofacial Orthop 2003; 124: 593-599.

54. Wiechmann D, Schwestka-Polly R, Hohoff A. Herbst appliance in lingual orthodontics. Am J Orthod Dentofacial Orthop 2008; 134: 439-446.

55. Lauren M, McIntyre F. A new computer-assisted method for design and fabrication of occlusal splints. Am J Orthod Dentofacial Orthop 2008; 133 (4 Suppl): S130-135.

56. Salmi M, Paloheimo KS, Tuomi J, et al. A digital process for additive manufacturing of occlusal splints: a clinical pilot study. J R Soc Interface 2013; 10: 20130203.

57. Antoszewska J, Papadopoulos MA, Park HS, Ludwig B. Five-year experience with orthodontic miniscrew implants: a retrospective investigation of factors influencing success rates. Am J Orthod Dentofacial Orthop 2009; 136: 158:e1-10.

58. Kravitz ND, Kusnoto B, Hohlt WF. A simplified stent for anterior miniscrew insertion. J Clin Orthod 2007; 41: 224-226.

59. Liu H, Liu DX, Wang G, et al. Accuracy of surgical positioning of orthodontic miniscrews with a computer - aided design and manufacturing template. Am J Orthod Dentofacial Orthop 2010; 6: 728e1-728e10.

60. Suzuki EY, Suzuki B. Accuracy of miniscrew implant placement with a 3-dimensional surgical guide. J Oral Maxillofacial Surg 2008; 6: 1245-1252.

61. Wang YT, Yu JH, Lo LJ, et al. Developing customized dental miniscrew surgical template from thermoplastic polymer material using image superimposition, CAD system and 3D printing. Biomed Res Int 2017; 2017: 1906197.

62. Sherwood KH, Burch J, Thompson W. Intrusion of supererupted molars with titanium miniplate anchorage. Angle Orthod 2003; 73: 597-601.

63. Tsui WK, Chua HDP, Cheung LK. Bone anchor systems for orthodontic application: a systematic review. Int J Oral Maxillofac Surg 2008; 41: 1427-1438.

64. Hourfar J, Kanavakis G, Goellner P, Ludwig B. Fully customized placement of orthodontic miniplates: a novel clinical technique. Head Face Med 2014; 10: 14 